\title{
Bu open Comparing two types of macrolide antibiotics for the purpose of assessing population-based drug interactions
}

\author{
Jamie L Fleet, ${ }^{1}$ Salimah Z Shariff, ${ }^{1,2}$ David G Bailey, ${ }^{3}$ Sonja Gandhi, ${ }^{1,4}$ \\ David N Juurlink, ${ }^{2,5,6}$ Danielle M Nash, ${ }^{1,2}$ Muhammad Mamdani, ${ }^{2,6,7}$ Tara Gomes, ${ }^{2}$ \\ Amit M Patel, ${ }^{1}$ Amit $X$ Garg $^{1,2,4}$
}

To cite: Fleet JL, Shariff SZ, Bailey DG, et al. Comparing two types of macrolide antibiotics for the purpose of assessing population-based drug interactions. BMJ Open 2013;3:e002857.

doi:10.1136/bmjopen-2013002857

- Prepublication history and additional material for this paper is available online. To view these files please visit the journal online (http://dx.doi.org/10.1136/ bmjopen-2013-002857)

Received 8 March 2013 Revised 24 May 2013

Accepted 14 June 2013

This final article is available for use under the terms of the Creative Commons Attribution Non-Commercial 3.0 Licence; see http://bmjopen.bmj.com

For numbered affiliations see end of article.

Correspondence to Dr Amit Garg;

amit.garg@|hsc.on.ca

\section{ABSTRACT}

Objective: Clarithromycin strongly inhibits enzyme cytochrome P450 3A4, preventing the metabolism of some other drugs, while azithromycin is a weak inhibitor. Accordingly, blood concentrations of other drugs increase with clarithromycin coprescription leading to adverse events. These macrolide antibiotics also differ on other properties that may impact outcomes. In this study, we compared outcomes in two groups of macrolide antibiotic users in the absence of potentially interacting drugs.

Design: Population-based retrospective cohort study. Setting: Ontario, Canada, from 2003 to 2010. Patients: Patients (mean 74 years) prescribed clarithromycin $(n=52251)$ or azithromycin (referent group, $\mathrm{n}=46618$ ).

Main outcomes: The primary outcomes were hospital admission within 30 days of a new antibiotic prescription with any of the 12 conditions examined separately (acute kidney injury, acute myocardial infarction, neuroimaging (proxy for delirium), hypotension, syncope, hyperkalaemia, hyponatraemia, hyperglycaemia, arrhythmia, ischaemic stroke, gastrointestinal bleeding and sepsis). The secondary outcome was mortality.

Results: The baseline characteristics of the two groups, including patient demographics, comorbid conditions, infection type and prescribing physician specialty, were nearly identical. The median daily dose was $1000 \mathrm{mg}$ for clarithromycin and $300 \mathrm{mg}$ for azithromycin and the median duration of dispensing antibiotics was 10 and 5 days, respectively. There was no difference between the groups in the risk of hospitalisation for any condition studied (relative risk ranged from 0.67 to 1.23 ). Compared with azithromycin, clarithromycin was associated with a slightly higher risk of all-cause mortality $(0.46 \%$ vs $0.37 \%$, relative risk $1.25,95 \% \mathrm{Cl} 1.03$ to 1.52 ).

Conclusions: Clarithromycin can be used to assess drug interactions in population-based studies with azithromycin serving as a control group. However, any differences in mortality observed between the two antibiotic groups in the setting of other drug use may be partially attributable to factors beyond the inhibition of drug metabolising enzymes and transporters, as the difference for this outcome was significant.

\section{ARTICLE SUMMARY}

\section{Article focus}

- This study describes the differences in adverse outcomes when either clarithromycin or azithromycin is prescribed in the absence of interacting drugs.

- Knowledge of the underlying differences between these two drugs is important for the interpretation of population-based drug-drug interaction studies.

\section{Key messages}

- There were no significant differences between clarithromycin and azithromycin on 12 hospitalisation outcomes; however, clarithromycin was associated with a slightly higher risk of all-cause mortality.

- Since there is no difference between clarithromycin and azithromycin in hospitalisation outcomes in the absence of interacting drugs, the use of azithromycin as a reference group is appropriate in drug-drug interaction studies.

- Most outcomes from drug-drug interaction studies can be attributed to the interaction rather than the underlying differences in these macrolide antibiotics.

Strengths and limitations of this study

- This is the first population-based study to compare outcomes between clarithromycin and azithromycin while excluding interacting drugs.

- Our large sample size allowed greater precision around the estimates reported and is representative of the province of Ontario as a whole.

- Further studies examining differences in all-cause mortality between the two antibiotics as well as non-macrolide antibiotics are warranted.

\section{INTRODUCTION}

Certain medication combinations can lead to altered pharmacokinetics that result in a higher systemic concentration of the drugs and accompanying greater risk of toxicity. ${ }^{1}$ The commonly used macrolide antibiotic clarithromycin can inhibit the drug metabolising 
enzyme cytochrome P450 3A4 (CYP3A4), as well as the Organic Anion Transporting Polypeptide transporters 1B1 (OATP1B1) and OATP1B3. ${ }^{2}$ These transporters and enzyme are present in the liver and small intestine, and about half of all the medications used today are affected by their processes. ${ }^{3}$ These include many types of statins, antiepileptics and antipsychotics. ${ }^{4-6}$ Interestingly, another macrolide antibiotic, azithromycin, is prescribed for similar indications and in comparable patients as clarithromycin, but unlike clarithromycin, it is only a very weak inhibitor of this enzyme and transporters. ${ }^{7-9}$ Thus, there is a growing interest in conducting population-based studies examining two groups of individuals newly prescribed either clarithromycin or azithromycin, where all patients are also chronically using another drug, such as a statin, which may interact with clarithromycin. ${ }^{10}$ The outcomes of the two groups can then be compared (with the azithromycin users acting as a control group) to assess the outcomes attributable to the clarithromycin-statin interaction. $^{10}$

However, as per the prescribing references, the two macrolide antibiotics do differ on the total daily dose and the recommended duration of therapy to treat infection which may influence compliance, as a dose would be more likely to be missed if taken over a longer period of time. ${ }^{11}{ }^{12}$ It is possible that these, and other properties of macrolide antibiotics, also impact patient outcomes. We wanted to be assured that outcomes observed in population-based drug interaction studies of clarithromycin compared with azithromycin are most likely attributable to the drug interaction being studied rather than other inherent differences between the two macrolide antibiotics. ${ }^{10}{ }^{13-15}$ For example, we recently published a study assessing statin and macrolide drug interactions, and noted that older patients coprescribed clarithromycin were more likely to be hospitalised with acute kidney injury in the subsequent 30 days compared with older patients coprescribed azithromycin. ${ }^{10}$ Observing an increase in the risk of acute kidney injury with clarithromycin versus azithromycin in the presence of a statin, but not in the absence of a statin, would provide additional evidence of statin toxicity from clarithromycin. ${ }^{10}$ The purpose of the current populationbased study was to compare the incidence of serious adverse events for two groups of older patients either prescribed clarithromycin or azithromycin in the absence of other drugs with metabolism potentially impacted by clarithromycin.

\section{METHODS}

\section{Setting and design}

All residents of the province of Ontario, Canada have universal access to hospital care and physician services. Individuals 65 years of age or older (approximately 2 million individuals in Ontario in 2012) also have universal prescription drug coverage. ${ }^{16}$ All healthcare encounters are prospectively recorded in health administrative databases, which are available for evaluation at the Institute for Clinical Evaluative Sciences in Ontario, Canada. We conducted a population-based retrospective cohort study using these large linked healthcare databases. We focused on adults over the age of 65 years, given their risk of drug toxicity and the availability of prescription data. We conducted this study according to a prespecified protocol that was approved by the research ethics board at Sunnybrook Health Sciences Centre (Toronto, Canada). The reporting of this study followed guidelines for observational studies (detailed in online supplementary appendix A). ${ }^{17}$

\section{Data sources}

We ascertained drug use, covariate information and outcome data using records from five administrative databases. Outpatient prescription drug information including the dispensing date, quantity of pills and number of days supplied is accurately recorded in the Ontario Drug Benefit Plan database, with an error rate less than $1 \% .^{18}$ Detailed diagnosis and procedural information on all inpatient hospitalisations in Ontario are recorded in the Canadian Institute for Health Information Discharge Abstract Database. Up to 25 unique diagnosis codes (ie, codes for acute kidney injury or hyperkalaemia) can be assigned at discharge to each hospital stay. The Ontario Health Insurance Plan database contains all health claims for inpatient and outpatient fee-for-service physician services. The Ontario Registered Persons Database contains demographic and vital statistics information on all Ontario residents who have ever been issued a health card. We have previously used these four databases to research adverse drug events, health outcomes and health services. ${ }^{19-21}$ The databases were complete for all variables used in this study. We also used the Ontario Registrar General Database to assess the cause of death for patients who died during follow-up.

Codes used to assess comorbidities in the 5 years prior to the receipt of the relevant prescription are detailed in online supplementary appendix B. This Appendix contains both the International Classification of Diseases, 9th revision (ICD-9) and 10th revision (ICD-10) codes, as both were in use during the study period. Codes used to ascertain outcomes are detailed in online supplementary appendix $\mathrm{C}$ with information on code validity when available. This online supplementary appendix only contains ICD-10 codes as ICD-9 codes were no longer used in Canada after 31 March 2002.

\section{Patients}

We established a cohort of patients with new prescriptions for clarithromycin. Our comparison (referent) group consisted of patients with new azithromycin prescriptions. Erythromycin, another macrolide antibiotic that inhibits several metabolising enzymes, was not included in our study since the number of prescriptions dispensed during our study period was low. 
The date of antibiotic prescription served as the index date, which is the start time for follow-up. We accrued patients from June 2003 to December 2010. We excluded the following antibiotic users from analysis: (1) those in their first year of eligibility for prescription drug coverage (age 65) to avoid incomplete past medication records, (2) those who were discharged from hospital in the 2 days prior to and including the index date to ensure that we were studying new outpatient antibiotic prescriptions, (3) those who received a prescription for more than one type of antibiotic on the index date in order to compare mutually exclusive groups, (4) those with end-stage renal disease prior to the index date and (5) those who were taking other potential CYP3A4, OATP1B1 or OATP1B3 inhibitors or substrates 180 days prior to the index date (medications such as protease inhibitors, statins, antifungals and calcium channel blockers-see online supplementary appendix D for full list). ${ }^{22}{ }^{23}$ When there were multiple episodes of macrolide antibiotic use for a given patient over the study period, we only selected the first one. For exclusions and baseline characteristics, we identified comorbidities in 5 years prior to the index date and concurrent drug therapy in 180 days prior to the index date (see online supplementary appendix B).

\section{Outcomes}

All patients were followed for 30 days after the index date for the assessment of outcomes. We assessed hospital admissions involving any of the 12 medical conditions; each condition was examined separately: acute kidney injury, acute myocardial infarction, neuroimaging (CT head scan as a proxy for delirium), hypotension, syncope, hyperkalaemia, hyponatraemia, hyperglycaemia, arrhythmia, ischaemic stroke, gastrointestinal bleeding and sepsis. These conditions are potential adverse events when clarithromycin interferes with the pharmacokinetics of other drugs. For example, the use of clarithromycin with a calcium channel blocker may cause hypotension and acute kidney injury. ${ }^{15}{ }^{24-28} \mathrm{~A}$ small number of events in our population precluded analyses of three other conditions of interest: rhabdomyolysis, hypoglycaemia and neuroleptic malignant syndrome. We also assessed all-cause mortality.

There are up to 25 diagnostic codes that can be assigned per hospital admission; patients with multiple codes were accounted for under each outcome of interest. Wherever possible, we selected validated codes that performed well for identifying the conditions of interest (code lists and validations fully detailed in online supplementary appendix $\mathrm{C}$ ).

\section{Statistical analysis}

We compared baseline characteristics between new users of clarithromycin and azithromycin using standardised differences. ${ }^{29} \quad 30$ This metric describes differences between group means relative to the pooled SD and is considered to indicate a meaningful difference if it is greater than $10 \%$. The risk of developing an outcome was expressed in relative terms. We used multivariable logistic regression analyses to estimate ORs and 95\% CIs, adjusting for age (per year), sex and Charlson comorbidity score (a popular measure of comorbidity). ${ }^{31}$ We interpreted ORs as relative risks (appropriate given the incidences observed). We conducted all analyses with SAS V.9.2 (SAS Institute Incorporated, Cary, North Carolina, USA, 2008).

\section{RESULTS}

There were a total of 1958432 macrolide antibiotic prescriptions during our study period. Cohort selection is presented in online supplementary appendix E. After applying our exclusion criteria, including evidence of any interacting drug and restricting to the first antibiotic prescription per patient, 98869 patients remained: 52251 clarithromycin users and 46618 azithromycin users.

The baseline characteristics of the two groups with respect to comorbidities and use of other medications were nearly identical (table 1; all standardised differences between the groups were less than $3 \%$ ). For both groups, the median age was 71 years and $54 \%$ of patients were women. The cause of infection was recorded in some patients and appeared to be comparable between the two groups, as were the cultures and concurrent bronchodilators and steroid prescriptions around the time of the index date (table 1). The specialty of the prescribing physician, when available, was also comparable between the two groups (table 1).

Consistent with the drug prescribing references, the median daily dose was $1000 \mathrm{mg}$ for clarithromycin and $300 \mathrm{mg}$ for azithromycin. The median duration of dispensing antibiotics was 10 days for clarithromycin and 5 days for azithromycin. ${ }^{11} 12$

The outcome of hospitalisation with each of the 12 conditions examined separately is presented in table 2. Results are expressed with patients receiving azithromycin as the referent group. There were no significant differences between the clarithromycin and azithromycin groups on any of the 11 hospitalisation outcomes, and the relative risk ranged from 0.67 to 1.23 . Results were consistent across all adjusted analyses (table 2).

The results of all-cause mortality within 30 days of the antibiotic prescription are also presented in table 2 . Compared with azithromycin, clarithromycin was associated with a slightly higher risk of all-cause mortality ( $0.46 \%$ vs $0.37 \%$, relative risk $1.25,95 \%$ CI 1.03 to 1.52$)$.

After observing a difference in all-cause mortality between our groups, we considered the five most common causes of death (table 3). There were no significant differences in these causes of death between the two groups.

\section{DISCUSSION}

The contrasting outcomes of patients prescribed clarithromycin to those prescribed azithromycin in the 
Table 1 Baseline characteristics

\begin{tabular}{|c|c|c|c|}
\hline & Clarithromycin $n=52251$ & Azithromycin $n=46618$ & Standardised differences* \\
\hline \multicolumn{4}{|l|}{ Demographics } \\
\hline Age, years, median (IQR) & $71(68-77)$ & $71(68-77)$ & \\
\hline Women, n (\%) & 27932 (53.5) & $25682(55.1)$ & 0.03 \\
\hline \multicolumn{4}{|l|}{ Income quintile } \\
\hline First (lowest) & $8951(17.1)$ & 7706 (16.5) & 0.02 \\
\hline Second & $10447(20.0)$ & 8899 (19.1) & 0.02 \\
\hline Third (middle) & $10153(19.4)$ & 8937 (19.2) & 0.01 \\
\hline Fourth & $10822(20.7)$ & 9633 (20.7) & 0 \\
\hline Fifth (highest) & $11703(22.4)$ & $11285(24.2)$ & 0.04 \\
\hline \multicolumn{4}{|l|}{ Year of cohort entry†, n (\%) } \\
\hline 2003-2005 & 21369 (40.9) & $18979(40.7)$ & 0.01 \\
\hline 2006-2008 & $19236(36.8)$ & 17198 (36.9) & 0.01 \\
\hline 2009-2010 & 11646 (22.3) & $10441(22.4)$ & 0.01 \\
\hline \multicolumn{4}{|l|}{ Comorbidities, n (\%) } \\
\hline Cancer & $12733(24.4)$ & $11473(24.6)$ & 0.01 \\
\hline Chronic kidney disease $\ddagger$ & $644(1.2)$ & $566(1.2)$ & 0 \\
\hline Coronary artery disease $\S$ & $7531(14.4)$ & $6956(14.9)$ & 0.01 \\
\hline Diabetes mellitus & $855(1.6)$ & $816(1.8)$ & 0.01 \\
\hline Heart failure & $1656(3.2)$ & $1536(3.3)$ & 0.01 \\
\hline Peripheral vascular disease & $175(0.3)$ & $176(0.4)$ & 0.01 \\
\hline Stroke/transient ischaemic attack & $246(0.5)$ & $249(0.5)$ & 0.01 \\
\hline \multicolumn{4}{|c|}{ Medication use in the prior 6 months, $\mathrm{n}(\%)$} \\
\hline ACE inhibitors or ARBs & $2769(5.3)$ & $2543(5.5)$ & 0.01 \\
\hline$\beta$ blockers & $1787(3.4)$ & $1720(3.7)$ & 0.01 \\
\hline Potassium sparing diuretics & $461(0.9)$ & $389(0.8)$ & 0.01 \\
\hline Loop diuretics & $103(0.2)$ & $120(0.3)$ & 0.01 \\
\hline NSAIDs (excluding ASA) & $2483(4.8)$ & $2389(5.1)$ & 0.02 \\
\hline Thiazide diuretics & $3479(6.7)$ & $3171(6.8)$ & 0.01 \\
\hline \multicolumn{4}{|l|}{ Cause of infection, $\mathrm{n}(\%)$} \\
\hline Genitourinary infection & $261(0.5)$ & $265(0.6)$ & 0.01 \\
\hline Oropharyngeal infection & 839 (1.6) & $1,000(2.1)$ & 0.04 \\
\hline Respiratory infection & $22084(42.3)$ & $17503(37.5)$ & 0.10 \\
\hline Sinus infection & $4,000(7.7)$ & $3,178(6.8)$ & 0.03 \\
\hline Skin infection & 659 (1.3) & $320(0.7)$ & 0.06 \\
\hline Missing & $27843(53.3)$ & 22266 (47.8) & 0.11 \\
\hline \multicolumn{4}{|l|}{ Cultures $^{* *}, \mathrm{n}(\%)$} \\
\hline Blood & $28(0.1)$ & $21(0.0)$ & 0 \\
\hline Genitourinary & $26(0.0)$ & $69(0.01)$ & 0.03 \\
\hline Gynaecology & $120(0.2)$ & $134(0.3)$ & 0.01 \\
\hline Sputum & $127(0.2)$ & $75(0.2)$ & 0.02 \\
\hline Urine & $1,090(2.1)$ & $931(2.0)$ & 0.01 \\
\hline \multicolumn{4}{|c|}{ Concurrent medication prescription, n (\%) } \\
\hline Inhaled steroids & $28(0.1)$ & $31(0.1)$ & 0.01 \\
\hline Bronchodilators & $1202(2.3)$ & $929(2.0)$ & 0.02 \\
\hline \multicolumn{4}{|c|}{ Main specialty of prescribing physician, $\mathrm{n}(\%)$} \\
\hline GP/FP & $39743(76.1)$ & 34308 (73.6) & 0.06 \\
\hline Internal medicine & $280(0.5)$ & $260(0.6)$ & 0.01 \\
\hline General surgery & $100(0.2)$ & $151(0.3)$ & 0.02 \\
\hline Other & $1148(2.2)$ & $1042(2.2)$ & 0 \\
\hline Missing & $10980(21.0)$ & $10857(23.3)$ & 0.06 \\
\hline
\end{tabular}

Data presented as number (per cent), except for age, which is presented as mean (SD).

*Standardised differences are less sensitive to sample size than traditional hypothesis tests. They provide a measure of the difference between groups divided by the pooled SD; a value greater than $10 \%(0.1)$ is interpreted as a meaningful difference between the groups. †The year of cohort entry is also referred to as the index date.

$\ddagger$ Assessed by administrative database codes.

$\S$ Coronary artery disease includes the receipt of coronary artery bypass graft surgery, percutaneous coronary intervention and diagnoses of angina.

ПAssessed by the receipt of insulin or oral antihyperglycaemics.

${ }_{\star \star *}$ Cultures recorded within 2 weeks prior to and 1 week after the index date.

ARB, angiotensin II receptor blocker; FP, family practitioner; GP, general practitioner; NSAID, non-steroidal anti-inflammatory. 
Table 2 Hospitalisations with various conditions and all-cause mortality

\begin{tabular}{|c|c|c|c|c|}
\hline & \multicolumn{2}{|c|}{ Number of events (\%)* } & \multirow[b]{2}{*}{$\begin{array}{l}\text { Unadjusted relative risk } \\
(95 \% \mathrm{Cl})\end{array}$} & \multirow[b]{2}{*}{$\begin{array}{l}\text { Adjusted relative risk } \\
(95 \% \mathrm{Cl}) \dagger\end{array}$} \\
\hline & $\begin{array}{l}\text { Clarithromycin } \\
n=52251\end{array}$ & $\begin{array}{l}\text { Azithromycin } \\
n=46618\end{array}$ & & \\
\hline Acute kidney injury & $52(0.10)$ & $44(0.09)$ & $1.05(0.71$ to 1.58$)$ & $1.06(0.71$ to 1.58$)$ \\
\hline Myocardial infarction & $39(0.07)$ & $30(0.06)$ & $1.16(0.72$ to 1.87$)$ & $1.15(0.71$ to 1.85$)$ \\
\hline Neuroimaging $\ddagger$ & $582(1.11)$ & $496(1.06)$ & 1.05 (0.93 to 1.18$)$ & 1.04 (0.93 to 1.18$)$ \\
\hline Hypotension & $19(0.04)$ & $14(0.03)$ & 1.21 (0.61 to 2.42$)$ & 1.21 (0.61 to 2.41$)$ \\
\hline Syncope & $14(0.03)$ & $12(0.03)$ & $1.04(0.48$ to 2.25$)$ & $1.04(0.48$ to 2.25$)$ \\
\hline Hyperkalaemia & $9(0.02)$ & $12(0.03)$ & $0.67(0.28$ to 1.59$)$ & $0.67(0.28$ to 1.60$)$ \\
\hline Hyponatraemia & $29(0.06)$ & $29(0.06)$ & 0.89 (0.53 to 1.49$)$ & $0.90(0.54$ to 1.51$)$ \\
\hline Hyperglycaemia & $22(0.04)$ & $16(0.03)$ & 1.23 (0.64 to 2.34$)$ & 1.22 (0.64 to 2.33$)$ \\
\hline Arrhythmia & 49 (0.09) & $52(0.11)$ & $0.84(0.57$ to 1.24$)$ & 0.84 (0.57 to 1.24$)$ \\
\hline Ischaemic stroke & $17(0.03)$ & $16(0.3)$ & 0.95 (0.48 to 1.88$)$ & $0.94(0.47$ to 1.86$)$ \\
\hline Gastrointestinal bleeding & $32(0.06)$ & $30(0.06)$ & 0.95 (0.58 to 1.57$)$ & 0.95 (0.58 to 1.56$)$ \\
\hline Sepsis & $28(0.05)$ & $18(0.04)$ & 1.39 (0.77 to 2.51$)$ & 1.38 (0.76 to 2.49$)$ \\
\hline All-cause mortality & $241(0.46)$ & $172(0.37)$ & 1.25 (1.03 to 1.52$)$ & $1.27(1.04$ to 1.55$)$ \\
\hline \multicolumn{5}{|c|}{$\begin{array}{l}\text { Patients prescribed azithromycin served as the comparator group. } \\
\text { *The number of events (and the proportion of patients who experienced an event) for all outcomes except all-cause mortality was assessed } \\
\text { by hospital diagnosis codes. For some outcomes, this underestimates the true event rate because these codes have high specificity but low } \\
\text { sensitivity. } \\
\text { †Adjusted for three covariates: age, sex and Charlson comorbidity score. } \\
\text { †Neuroimaging consisted of codes for Cl head scan as a proxy for delirium. }\end{array}$} \\
\hline
\end{tabular}

presence of a drug with metabolism potentially impacted by clarithromycin present a potentially attractive method of assessing population-based clarithromycin drug interactions in routine care. However, these two macrolide antibiotics also differ on other properties besides their inhibition of drug metabolising enzymes and transporters that may impact patient outcomes. In this study, we compared the baseline characteristics and outcomes of patients prescribed either clarithromycin or azithromycin in the absence of potentially interacting drugs. The two groups did not differ in patient baseline demographics, comorbid characteristics, the type of infection or the specialty of the prescribing physician. In other words, the two drugs appeared to be used for similar indications and demonstrated similar clinical usage patterns. With respect to the study outcomes,

Table 3 Deaths due to the following causes

\begin{tabular}{|c|c|c|}
\hline & \multicolumn{2}{|c|}{ Number of events $(\%)^{\star}$} \\
\hline & $\begin{array}{l}\text { Clarithromycin } \\
n=52251\end{array}$ & $\begin{array}{l}\text { Azithromycin } \\
n=46618\end{array}$ \\
\hline $\begin{array}{l}\text { Disease of the } \\
\text { circulatory system }\end{array}$ & $64(0.12)$ & $50(0.11)$ \\
\hline Neoplasm & $48(0.09)$ & $32(0.07)$ \\
\hline $\begin{array}{l}\text { Disease of the } \\
\text { respiratory system }\end{array}$ & $35(0.07)$ & $32(0.07)$ \\
\hline Mental disorder & $28(0.05)$ & $13(0.03)$ \\
\hline $\begin{array}{l}\text { Disease of the nervous } \\
\text { system }\end{array}$ & $25(0.05)$ & $13(0.03)$ \\
\hline Other & $41(0.08)$ & $32(0.07)$ \\
\hline
\end{tabular}

there were no differences between the two groups on any of the 12 hospitalisation conditions that we studied.

Overall, these results support the utility of macrolide antibiotics to assess population-based drug interactions for the hospital conditions presented in this report. This is particularly true when conducting studies in settings where the observed results are consistent with medications known to have potential for drug-drug interactions based on pharmacokinetic data and case reports. For example, a high blood concentration of some statins is realised when taken concurrently with clarithromycin, as the latter inhibits the CYP3A4 enzyme responsible for statin metabolism. ${ }^{10}$ This can lead to rhabdomyolysis and acute kidney injury. In the present study, in the absence of statin use, there was no difference in hospitalisation with acute kidney injury between the two macrolide antibiotic groups. Thus, there is more assurance that the outcomes observed in the aforementioned study of clarithromycin coprescribed with a statin are attributable to the interaction between the drugs.

In the present study, there was a small absolute difference in all-cause mortality with clarithromycin compared with azithromycin, without any clear difference in the cause of death. While this may be a chance finding, it is also possible that there may be inherent differences in the use or nature of these two antibiotics that impact mortality. Consistent with the drug prescribing references, the median duration of antibiotic treatment was higher with clarithromycin compared with azithromycin. Additionally, differences in daily dose and day supply between the two macrolide antibiotics were found, and there could be differences in the frequency of dose. As clarithromycin is taken twice a day for the duration of therapy, unlike azithromycin, there could be differences 
in drug adherence. Other differences exist; for example, azithromycin is less bioavailable than clarithromycin, especially when taken with food. ${ }^{32}$ On the other hand, clarithromycin is transformed into an active metabolite, where most other macrolide antibiotics are not. ${ }^{33}$ For these reasons, some of the association between macrolide antibiotic type and mortality may partially be attributable to factors beyond the inhibition of drug transporters and metabolising enzymes, although it too may not be reflective of a difference between the drugs at all. It may also be useful to determine if the magnitude of the association observed in the present study differs with associations observed in other drug-drug interaction studies, using statistical tests of interaction (such as the Bland-Altman test on the two sets of results). ${ }^{34}$ Additionally, in the future, studies with other non-macrolide antibiotics, compared with clarithromycin, may be warranted, as macrolide antibiotics have a higher rate of mortality as they are potentially arrhythmogenic. $^{35-37}$

Our study has a number of strengths. This study was carried out in the province of Ontario where residents have the benefit of universal healthcare for all citizens and a province-wide drug plan for older adults, with this information accessible for study purposes. Accordingly, there were a large number of patients accrued into our study, which provided reasonable precision for the outcomes that are reported. The large sample size also provided adequate data to reasonably compare clarithromycin and azithromycin on the baseline characteristics and patterns of clinical use.

Our study does have some limitations. Despite the large sample size, we had very few events to meaningfully look at some outcomes such as rhabdomyolysis, neuroleptic malignant syndrome and hypoglycaemia. For reasons of privacy, we are not permitted to report information for small cell sizes which also precluded meaningful analysis of some types of cause of death, such as infectious disease. Drug-drug interactions at the population level in routine care are complex and understudied. While we took a comprehensive approach to exclude interacting drugs, it is still possible that interactions with other drugs may have occurred. The efficacy of pathogen eradication is similar between the two macrolides for some illnesses, but was not formally assessed here. ${ }^{38} 39$ Finally, because our hospital-based outcomes were assessed using hospital diagnosis codes (which have limited sensitivity for some outcomes), rather than prospective data collection, we most likely underestimated the true event rate of the outcomes. However, because the outcomes were assessed no differently between the clarithromycin and azithromycin groups, we do not anticipate that this biased our relative measures of risk.

\section{CONCLUSION}

In conclusion, we have established that patterns of use and common clinical outcomes do not differ appreciably between clarithromycin and azithromycin, suggesting that clarithromycin may be a useful medication to assess drug-drug interactions in populationbased studies with azithromycin serving as the control group. If in future drug-drug interaction studies, differences in mortality between groups of patients prescribed each of the two antibiotics exist, it should be noted that some of the association may be attributable to factors unrelated to the enzyme metabolism of the drugs.

\section{Author affiliations}

${ }^{1}$ Division of Nephrology, Department of Medicine, Western University, London, Canada

${ }^{2}$ Institute for Clinical Evaluative Sciences, Ontario, Canada

${ }^{3}$ Lawson Health Research Institute, London Health Sciences Centre, London, Canada

${ }^{4}$ Department of Epidemiology and Biostatistics, Western University, London, Canada

${ }^{5}$ Division of Clinical Pharmacology, Department of Medicine, University of Toronto, Toronto, Canada

${ }^{6}$ Departments of Health Policy, Management, and Evaluation, University of Toronto, Toronto, Canada

${ }^{7}$ Leslie Dan Faculty of Pharmacy, University of Toronto, Toronto, Canada

Acknowledgements We would like to thank Brogan Inc, Ottawa for use of its Drug Product and Therapeutic Class Database. We thank the late Dr Milton Haines, Ms Barbara Jones, Mr Jeff Lamond and others from Gamma Dynacare for their use of the outpatient laboratory database. We also thank Mr Glen Kearns from the London Health Sciences Centre who facilitated the use of linked hospital laboratory databases.

Contributors JLF participated in the co-ordination of the study, study design, provided interpretation of the study results and drafted the manuscript. SZS participated in the study design, performed the analysis and provided interpretation of the study results. DGB, SG and DNJ participated in the study design and provided drug information and feedback on the manuscript. DMN, MM, TG and AMP participated in the study design and provided feedback on the manuscript. AXG conceived of the study, participated in its design and interpretation, helped draft the manuscript and provided feedback on the manuscript. All authors read and approved the final manuscript.

Funding This project was conducted at the Institute for Clinical Evaluative Sciences (ICES)@Western Site. ICES is funded by an annual grant from the Ontario Ministry of Health and Long-term Care. ICES@Western is funded by operating grants from the Academic Medical Organization of Southwestern Ontario, the Schulich School of Medicine and Dentistry and the Lawson Health Research Institute.

Competing interests None.

Provenance and peer review Not commissioned; externally peer reviewed.

Data sharing statement No additional data are available.

\section{REFERENCES}

1. Dresser GK, Spence JD, Bailey DG. Pharmacokineticpharmacodynamic consequences and clinical relevance of cytochrome P450 3A4 inhibition. Clin Pharmacokinet 2000;38:41-57.

2. Wilkinson GR. Drug metabolism and variability among patients in drug response. N Engl J Med 2005;352:2211-21.

3. Bailey DG, Dresser G, Arnold JM. Grapefruit-medication interactions: forbidden fruit or avoidable consequences? CMAJ 2013;185:309-16.

4. Hutson JR, Fischer HD, Wang X, et al. Use of clarithromycin and adverse cardiovascular events among older patients receiving donepezil: a population-based, nested case-control study. Drugs Aging 2012;29:205-11.

5. Neuvonen PJ, Niemi M, Backman JT. Drug interactions with lipid-lowering drugs: mechanisms and clinical relevance. Clin Pharmacol Ther 2006;80:565-81. 
6. Pauwels O. Factors contributing to carbamazepine-macrolide interactions. Pharmacol Res 2002;45:291-8.

7. Seithel A, Eberl S, Singer K, et al. The influence of macrolide antibiotics on the uptake of organic anions and drugs mediated by OATP1B1 and OATP1B3. Drug Metab Dispos 2007;35:779-86.

8. Westphal JF. Macrolide-induced clinically relevant drug interactions with cytochrome P-450A (CYP) 3A4: an update focused on clarithromycin, azithromycin and dirithromycin. Br J Clin Pharmacol 2000;50:285-95.

9. Polasek TM, Miners JO. Quantitative prediction of macrolide drug-drug interaction potential from in vitro studies using testosterone as the human cytochrome P4503A substrate. Eur J Clin Pharmacol 2006;62:203-8.

10. Patel AM, Shariff S, Bailey DG, et al. Statin toxicity from macrolide antibiotic co-prescription: a population based study of older adults. Ann Intern Med 2013;158:869-76.

11. Clarithromycin Drug Information. UptoDate online V 20.3. 2012. 5-25-0120. Ref Type: Electronic Citation

12. Azithromycin Drug Information. UptoDate online V 20.3. 2012. 5-25-0120. Ref Type: Electronic Citation

13. McKinnell J, Tayek JA. Short term treatment with clarithromycin resulting in colchicine-induced rhabdomyolysis. J Clin Rheumatol 2009;15:303-5.

14. Pasqualetti G, Bini G, Tognini S, et al. Clarithromycin-induced rhabdomyolysis: a case report. Int J Gen Med 2012;5:283-5.

15. Wright AJ, Gomes T, Mamdani MM, et al. The risk of hypotension following co-prescription of macrolide antibiotics and calciumchannel blockers. CMAJ 2011;183:303-7.

16. Statistics Canada. Population by sex and age group, by province and territory. Ottawa: Statistics Canada, 2011; 8-10-0120. Ref Type: Electronic Citation

17. von EE, Altman DG, Egger M, et al. The Strengthening the Reporting of Observational Studies in Epidemiology (STROBE) statement: guidelines for reporting observational studies. Ann Intern Med 2007;147:573-7.

18. Levy AR, O'Brien BJ, Sellors C, et al. Coding accuracy of administrative drug claims in the Ontario Drug Benefit database. Can J Clin Pharmacol 2003;10:67-71.

19. Jain AK, Cuerden MS, McLeod I, et al. Reporting of the estimated glomerular filtration rate was associated with increased use of angiotensin-converting enzyme inhibitors and angiotensin-II receptor blockers in CKD. Kidney Int 2012;81:1248-53.

20. Shih AW, Weir MA, Clemens KK, et al. Oral bisphosphonate use in the elderly is not associated with acute kidney injury. Kidney Int 2012;82:903-8.

21. Zhao $Y Y$, Weir MA, Manno M, et al. New fibrate use and acute renal outcomes in elderly adults: a population-based study. Ann Intern Med 2012;156:560-9.

22. Flockhart DA. Drug interactions: cytochrome P450 drug interaction table. India. 2012. Ref Type: Electronic Citation
23. Niemi M, Pasanen MK, Neuvonen PJ. Organic anion transporting polypeptide 1B1: a genetically polymorphic transporter of major importance for hepatic drug uptake. Pharmacol Rev 2011:63:157-81.

24. Calcium channel blockers+macrolides: elderly patients hospitalised for low blood pressure. Prescrire Int 2012;21:182.

25. Bode C. The nasty surprise of a complex drug-drug interaction. Drug Discov Today 2010;15:391-5.

26. Lee CY, Marcotte F, Giraldeau G, et al. Digoxin toxicity precipitated by clarithromycin use: case presentation and concise review of the literature. Can J Cardiol 2011;27:870-6.

27. Trieu J, Emmett L, Perera C, et al. Rhabdomyolysis resulting from interaction of simvastatin and clarithromycin demonstrated by Tc-99m MDP scintigraphy. Clin Nucl Med 2004;29:803-4.

28. Kute VB, Shah PR, Goplani KR, et al. Successful treatment of refractory hypotension, noncardiogenic pulmonary edema and acute kidney injury after an overdose of amlodipine. Indian J Crit Care Med 2011;15:182-4

29. Austin PC. Using the standardized difference to compare the prevalence of a binary variable between two groups in observational research. Commun Stat Simulation Comput 2009;38:1228-34.

30. Mamdani M, Sykora K, Li P, et al. Reader's guide to critical appraisal of cohort studies: 2. Assessing potential for confounding. BMJ 2005;330:960-2.

31. Charlson ME, Pompei P, Ales KL, et al. A new method of classifying prognostic comorbidity in longitudinal studies: development and validation. J Chronic Dis 1987;40:373-83.

32. Kanatani MS, Guglielmo BJ. The new macrolides. Azithromycin and clarithromycin. West J Med 1994;160:31-7.

33. Davey PG. The pharmacokinetics of clarithromycin and its $14-\mathrm{OH}$ metabolite. J Hosp Infect 1991;19(Suppl A):29-37.

34. Bland JM, Altman DG. Statistical methods for assessing agreement between two methods of clinical measurement. Lancet 1986;1:307-10.

35. Ray WA, Murray KT, Meredith S, et al. Oral erythromycin and the risk of sudden death from cardiac causes. $N$ Engl J Med 2004;351:1089-96.

36. Ray WA, Murray KT, Hall K, et al. Azithromycin and the risk of cardiovascular death. N Engl J Med 2012;366:1881-90.

37. Zambon A, Polo FH, Contiero P, et al. Effect of macrolide and fluoroquinolone antibacterials on the risk of ventricular arrhythmia and cardiac arrest: an observational study in Italy using case-control, case-crossover and case-time-control designs. Drug Saf 2009;32:159-67.

38. Bradbury F. Comparison of azithromycin versus clarithromycin in the treatment of patients with lower respiratory tract infection. $J$ Antimicrob Chemother 1993;31(Suppl E):153-62.

39. Muller O. Comparison of azithromycin versus clarithromycin in the treatment of patients with upper respiratory tract infections. J Antimicrob Chemother 1993;31(Suppl E):137-46. 